\title{
Source of variation on lingual vibrotactile thresholds: II. Adequate experimenter training
}

\author{
DONALD FUCCI, LINDA PETROSINO, JAMES CANTRELL, \\ and NEAL SLOANE \\ Ohio University, Athens, Ohio 45701
}

\begin{abstract}
Vibrotactile thresholds were obtained from the anterior midline of the tongue for 10 randomly selected normal adults. Results were subsequently compared with an initial set of data obtained from three different examiners with varying degrees of exposure to threshold assessment technique. The examiner for this study was the inexperienced examiner from the initial investigation, who obtained significantly poorer threshold results than the other two experienced examiners. The purpose of this investigation was, therefore, to further explore what constituted adequate lingual vibrotactile threshold training for the novice examiner. Results of statistical analysis revealed that further practice on the part of the inexperienced examiner in threshold testing led to threshold values that were similar to those of the original experienced examiners. Implications of adequate experimenter training are discussed.
\end{abstract}

The term "lingual vibrotactile threshold" refers in theory to the faintest detectable stimulus that can be felt on the tongue using vibration as the stimulus mode. Even though this sounds simple enough, the experimenter must consider a number of variables that may affect the stability of threshold measurement. An early concern was for possible variation in the subject population being tested. Telage, Fucci, and Arnst (1972) looked at intersubject variability of lingual vibrotactile thresholds obtained from a group of 110 normal-speaking adults. Data collected from two different test frequencies reflected little intersubject variability. Another factor to be considered was the measurement technique. Fucci, Arnst, and Telage (1972) compared ascending-pulsed, ascending-continuous, and descending-continuous stimulus presentations across 52 subjects. The ascendingpulsed condition permitted the lowest threshold values to be obtained, whereas the highest threshold results were obtained from the descending-continuous condition. A third area of consideration was equipment influences on vibrotactile thresholds. Fucci, Crary, and Telage (1977), Telage, Fucci, and Crary (1977), and Telage and Petrosino (1978) investigated lingual vibrotactile thresholds obtained using different tongueclamping procedures. Combined results showed lower thresholds using a solid lower disk as opposed to a ring disk. It was also determined that a frequency-specific response was to be obtained regardless of the clamping procedure used. However, when no clamp was used as a tongue support mechanism, a flat response across frequencies was reported. The above-mentioned investigations have shown that interactions can take place between instrumentation and methodology to alter threshold values.
Recently, Fucci, Petrosino, Sloane, and Cantrell (1981) investigated possible influences on threshold values produced from the experimenter himself. Lingual vibrotactile threshold data were obtained from three different experimenters with varying degrees of threshold assessment experience. Results indicated that the one experimenter with no threshold assessment experience with respect to any sensory system (auditory or tactile) obtained lingual vibrotactile threshold results that were significantly poorer than those of the experimenters who had experience in either vibrotactile or auditory threshold assessment. The results from this study demonstrated that adequate training of threshold assessment technique is critical for the acquisition of reliable vibrotactile thresholds. These results provided the impetus for the present study on the experimenter as a source of variation on lingual vibrotactile thresholds. The present study was designed to establish the criteria for adequate experimenter training.

\section{METHOD}

\section{Subjects}

Ten subjects were randomly selected from a group of 76 students from an introductory course in speech and hearing sciences at Ohio University. The subjects ranged in age from 19 to 27 years, with a mean age of 19 years. None of the subjects reported a present or past history of speech or hearing disorders or any known sensory and/or motor impairments.

\section{Apparatus}

The equipment used in this study has been described in previous publications, the most recent being a chapter on the state of the art (Fucci \& Crary, 1979). The stimulus unit included a sine-wave generator, a frequency counter, an electronic switchinterval timer, an amplifier, a 2 - $\mathrm{dB}$ step variable attenuator, and an electromagnetic minivibrator. The pulsed vibratory signal 
generated had a $50 \%$ duty cycle (on $.5 \mathrm{sec}$ and off $.5 \mathrm{sec}$ ), with a rise and decay time of $100 \mathrm{msec}$. The measurement unit consisted of an accelerometer, a cathode follower, a microphone amplifier, and a voltmeter. A narrow-band noise generator was used to present auditory masking at a 70-dB hearing threshold level to subjects through TDH-39 headphones.

\section{Procedure}

The first experiment involved threshold data collection from three different experimenters (Fucci et al., 1981). Experimenter $A$ had 4 years of lingual vibrotactile threshold data collection experience. Experimenter B had no experience in lingual vibrotactile threshold data collection but had experience in audiological threshold assessment. Experimenter $C$ had no experience in lingual vibrotactile threshold data collection or audiological threshold assessment. This sequel to the first experiment focused on Experimenter C, in order to determine if a doubling of his original population of 10 subjects would cause his threshold assessments to become statistically similar to those of the experimenters with experience in threshold assessment. With this goal in mind, the inexperienced experimenter was asked to obtain lingual vibrotactile thresholds on 10 more subjects, using the same subject training criteria and the same threshold data collection procedure as used in the previous experiment.

Prior to testing, subjects received training to acquaint them with the nature of the stimulus and to familiarize them with the testing apparatus. The subjects were trained to detect the stimulus on the tongue at a frequency of $250 \mathrm{~Hz}$. A training criterion requiring each subject to produce three threshold responses such that no two varied by more than $10 \mathrm{MV}$ was used as an index of adequate training. Subsequent to adequate training, standardized instructions were read to each subject during the testing session. Each subject was seated in an adjustable chair and asked to extend his tongue between two plastic sterilized disks. A hole in the top disk provided access of the probe to the anterior midline section of the dorsum of the tongue. The contactor area of the probe was $.128 \mathrm{~cm}^{2}$. To maintain constant pressure of the vibrator on the tongue for all subjects, the probe was lowered until a voltmeter recorded contact. The probe was then lowered $1 \mathrm{~mm}$ farther into the lingual surface.

Lingual vibrotactile thresholds were obtained at a $250 \mathrm{~Hz}$ test frequency, since that is within the range of frequencies to which the tongue is most sensitive (Fucci, Hall, \& Weiner, 1971). Each subject was required to respond by raising his hand as soon as he detected the pulsed vibratory stimulus. An ascending psychophysical method of limits was used. The mean of three millivolt readings was accepted as the lingual threshold and was later changed to displacement in microns, with the standard "g" formula for acceleration conversion.

\section{RESULTS AND DISCUSSION}

Data in microns of peak displacement were averaged for the subjects in the experimental test condition. The means and standard deviations for Experimenter $\mathrm{A}$ and Experimenter B from the first experiment and a mean and standard deviation for Experimenter $\mathrm{C}$ from this second experiment are shown in Table 1. The mean values were strikingly similar across experimenters and were not indicative of abnormal variability. The data were subjected to an analysis of variance for a completely randomized design to determine if significant differences existed between experimenters (Bruning \& Kintz, 1977). Table 2 shows that the main effect for experimenters was nonsignificant at the .05 level. The data from the
Table 1

Threshold Means (in Microns) and Standard Deviations for Experimenter A and Experimenter B from the First Experiment, and the Threshold Mean (in Mcirons) and Standard Deviation for Experimenter C from the Present Experiment

\begin{tabular}{crr}
\hline Experimenter & Mean & \multicolumn{1}{c}{ SD } \\
\hline A & 1.15 & .47 \\
B & 1.06 & 1.20 \\
C & .97 & .43 \\
\hline
\end{tabular}

Table 2

Summary Table for the Analysis of Variance

\begin{tabular}{lrrrr}
\hline \multicolumn{1}{c}{ Source } & SS & df & MS & F \\
\hline Total & 5.05 & 29 & & \\
Between Groups & .15 & 2 & .08 & $.44^{*}$ \\
Within Groups & 4.90 & 27 & .18 & \\
\hline
\end{tabular}

*Nonsignificant at .05.

first experiment revealed that Experimenter $\mathrm{C}$ (the inexperienced threshold examiner) acquired statistically significant poorer thresholds from his subjects than did either of the experienced examiners. These data are suggestive of the fact that experience in threshold testing is critical for the acquisition of reliable threshold values. The second experiment revealed that by assessing the thresholds of 10 more subjects (20 altogether), Experimenter C's values became statistically similar to those of the experienced examiners. This finding indicates that adequate experimenter training had occurred. Review of this two-part investigation indicates that the inexperienced examiner may in fact be a potential source of variance in the acquisition of accurate lingual vibrotactile threshold data, and therefore the adequately trained examiner is critical for reliable data collection. Continued research is always necessary to isolate sources of variance in the data acquisition of lingual vibrotactile thresholds. It is only with the identification of these sources that we can begin to understand the complex oral sensory system as it really exists and functions.

\section{REFERENCES}

Bruning, J. L., \& Kintz, B. L. Computational handbook of statistics. Glenview, Ill: Scott-Foresman, 1977.

Fucci, D., Arnst, D., \& Telage, K. The effects of pulsed and continuous stimulation on vibrotactile thresholds obtained from the tongue. Psychonomic Science, 1972, 29, 83-84.

Fucci, D., \& Crary, M. A. Oral vibrotactile sensation and perception: State of the art. In N. J. Lass (Ed.), Speech and language: Advances in basic research and practice. New York: Academic Press, 1979.

Fucci, D., Crary, M. A., \& Telage, K. Lingual clamping procedures for measuring oral vibrotactile thresholds: II. Effects of using a lower clamping disk. Bulletin of the Psychonomic Society, 1977, 10, 457-459.

Fucci, D., Hall, D., \& Weiner, F. Normative study of oral and nonoral structures using vibrotactile stimuli. Perceptual and Motor Skills, 1971, 33, 1099-1105. 
Fucci, D., Petrosino, L., Slonen, N., \& Cantrell, J. Source of variation on lingual vibrotactile thresholds: $I$. The influence of experimenter experience. Bulletin of the Psychonomic Society, 1981, 17, 231-232.

Telage, K., Fucci, D., \& Arnst, D. Normative study of oral vibrotactile sensitivity. Perceptual and Motor Skills, 1972, 35, 671-676.

Telage, K., Fucci, D., \& Crary, M. A. Lingual clamping procedures for measuring oral vibrotactile thresholds: I. Effects of using a free-surround disk. Bulletin of the Psychonomic Society, 1977, 10, 347-349.

Telage, K., \& Petrosino, L. Testing procedures for measuring oral vibrotactile thresholds: III. Effects obtained using a nonclamping method. Bulletin of the Psychonomic Society, 1978, 12, 291-293.

(Received for publication June 1, 1981.) 